ISSN 1112-9867

Available online at http://www.jfas.info

\title{
PHYTOECOLOGICAL STUDY OF TETRACLINIS ARTICULATA IN THE WOODLAND OF BENI AFFENE, SDAMAS CHERGUI (TIARET, ALGERIA)
}

\author{
M. E. Azzaoui*, M. Maatoug and M. Berrayah \\ Laboratoire d'Agro Biotechnologie et de Nutrition en Zones Semi Arides, Faculté des Science \\ de la Nature et de la vie, Université Ibn Khaldoun Tiaret, Algérie
}

Received: 26 November 2016 / Accepted: 24 April 2017 / Published online: 01 May 2017

\begin{abstract}
The Mediterranean flora is definitely considered as an exceptional diversity that deserves particular attention to be conserved. This work aims to quantify the floristic diversity of Tetraclinis articulata of Beni Affane located at the woodland of Sdamas Chergui (Tiaret, Algeria). The factorial analysis of correspondences allowed us to jointly process the floristic and ecological variables, and confirm that there is presently an original floristic facies of Tetraclinis articulata. This study area contains 13 families and 21 species, where the dominating biological type is Chamaephytics (52\%). We have described as well five frequent species: Tetraclinis articulata $33 \%$, Pistacia lentiscus $11 \%$, Globularia alypum $8 \%$, Phylleria angustifolia $9 \%$, Rosmarinus tournefortii $15 \%$.
\end{abstract}

Keywords: Tetraclinis articulata, Phytoecology, factorial analysis of correspondences, Beni Affane, Tiaret.

Author Correspondence, e-mail: moha-1500@ outlook.com

doi: http://dx.doi.org/10.4314/jfas.v9i2.23

\section{INTRODUCTION}

La couverture végétale constitue une des composantes principales des milieux naturels. La végétation joue également un rôle fondamental dans la structure et le fonctionnement de l'écosystème dont elle constitue une expression du potentiel biologique.

La flore du bassin méditerranéen est unanimement considérée comme étant d'une exceptionnelle diversité, et mérite à ce titre, une attention particulière pour sa conservation. 
Les principaux écosystèmes sont subdivisés selon la taille de ces végétaux, partant des forêts dites sclérophylles aux steppes en passant par les matorrals.

Les écosystèmes forestiers des monts de Beni Affene, situés au Nord-Ouest de la wilaya de Tiaret de l'Algérie occidentale, ont subi continuellement une régression due le plus souvent à une action conjuguée des facteurs climatiques et anthropiques.

Le couvert végétal est assez varié et y est dominé par les groupements à Tetraclinis articulata, qui représente environ $73 \%$ de la surface de Beni Affene et $1 \%$ de la superficie totale du Tetraclinis articulata en Algérie, qui est de l'ordre de 161000 ha [1].

Suite aux différents facteurs de dégradation notamment les incendies, l'aire de Tetraclinis articulata dans la zone d'étude a diminué et l'allure de la forêt se trouve constamment modifiée et transformée en matorrals plus ou moins denses. En effet, Plusieurs travaux sur les peuplements à Tetraclinis articulata réalisés en Afrique du nord en général et sur l'Algérie en particulier, confirment cette tendance notamment [1-4].

La forêt de Thuya est certainement le groupement végétal le plus caractéristique de l'étage semi-aride. Ces groupements forestiers et/ou pré forestiers présentent une proportion élevée de peuplements dégradés, dotés d'une capacité d'adaptation de réponse aux diverses pressions qu'ils subissent.

L'objectif de cette étude consiste à comprendre la diversité du couvert de la végétation qui constitue les groupements de Tetraclinis articulata sur les monts de Beni Affene (Wilaya de Tiaret, Algérie), et de caractériser l'état de l'écosystème et de mettre en évidence ses modifications naturelles résultent de la combinaison de processus géographique, climatique, et écologique mais aussi d'une emprise anthropique est omniprésente qui a façonné les paysages et leurs diversités.

\section{MATERIEL ET METHODES}

\subsection{Présentation de la zone d'étude}

La zone d'étude fait partie intégrante de la forêt domaniale de Beni Affane (massif du Sdamas Chergui). Elle est située au Nord-Ouest de la wilaya de Tiaret de l'Algérie occidentale. Du point de vue géographique, elle est située entre $35^{\circ} 17^{\prime}$ de latitude Nord et $1^{\circ} 03^{\prime}$ de longitude Est (figure 1). Elle s'étend sur une superficie de 4018 Has et regroupe 04 cantons, à savoir : Djbel Aref, Djbel Ghezala, Meska Et Tarlempt (tableau 1). 
Tableau 1. Répartition de la superficie de la forêt de Beni Affane selon les contons [5].

\begin{tabular}{|c|c|c|}
\hline Forêt & Noms des contons & Superficie (has) \\
\hline \multirow{3}{*}{ Beni Affane } & Djbel Aref & 896 \\
\hline & Djbel Ghezala & 1022 \\
\cline { 2 - 3 } & Meska & 1492 \\
\hline & Tarlempt & 608 \\
\hline
\end{tabular}
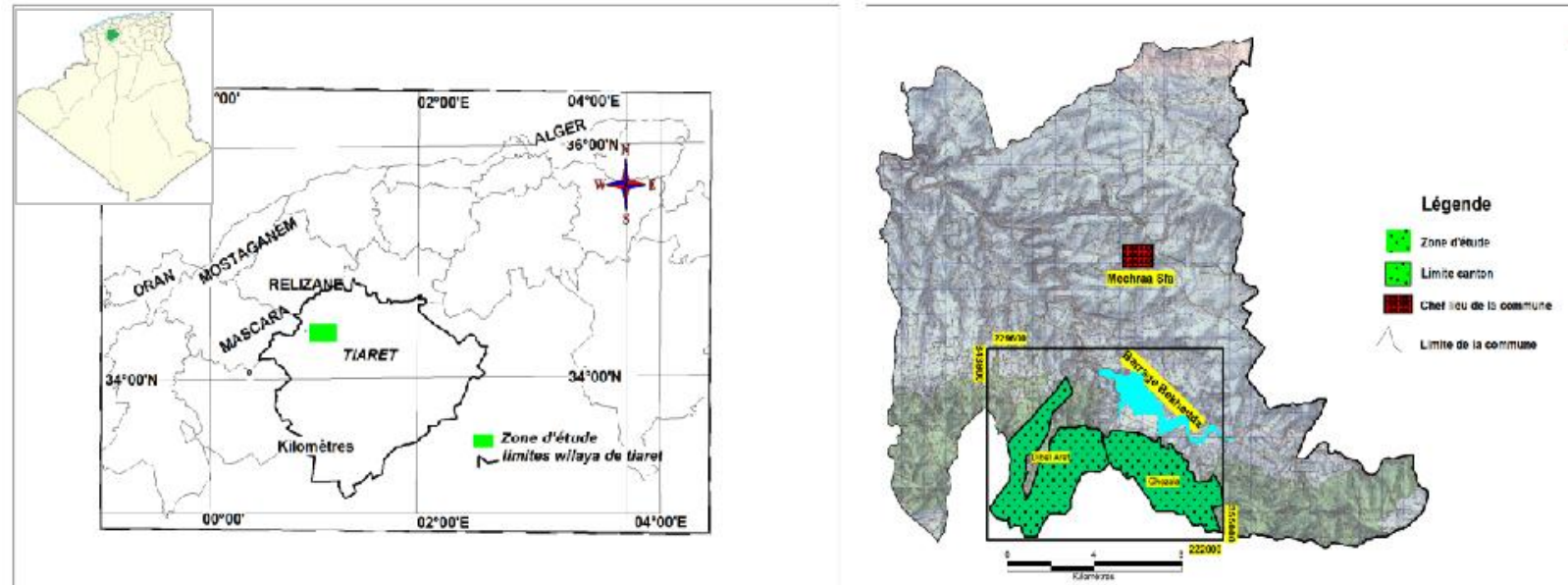

Fig.1. Situation géographique de la zone d'étude [5].

En effet, cette forêt est essentiellement occupée par le Tetraclinis articulata, qui est incontestablement l'espèce dominante comme le montre la figure 2. Toutefois et compte tenu des variations du milieu physique, notamment la topographie, la géologie, la climatologie, l'instabilité structurale du sol, certaines autres espèces peuvent être rencontrées telles que l'Olea europea var oleaster, Stipa tenassicima, Pistacia lentiscus, Phillyrea angustifolia, Globularia alypum, Quercus coccifira. 


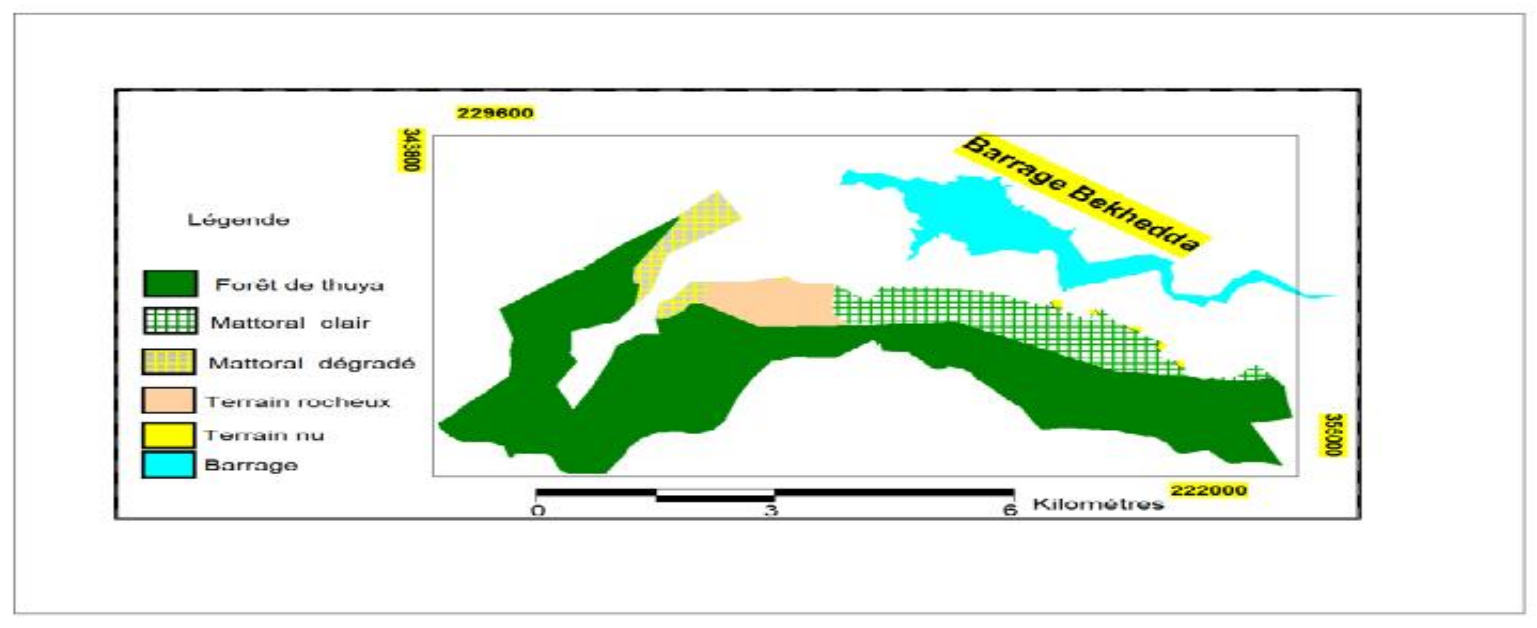

Fig.2. Carte d'occupation de sol [5].

Le relief est dans l'ensemble montagneux. Les altitudes varient entre $640 \mathrm{~m}$ et $1031 \mathrm{~m}$. Les pentes sont également fortes, les classes supérieures à $12,5 \%$ représentent plus de $41 \%$ du relief.

Les sols sont assez hétérogènes et leurs caractéristiques suivent la nature du substrat et la topographie. Ils peuvent être répartis en quatre classes : sols calcaires, sols rouges, sols peu évolués et les lithosols [6,19].

Le climat est de type méditerranéen avec un régime pluviométrique variable laissant apparaitre deux saisons bien distinctes, l'une pluvieuse qui s'étend de Septembre à Mai et l'autre chaude et sèche. Ceci exprime le contraste très important qui sévit durant l'année. La moyenne des précipitations oscillait entre 350 et $450 \mathrm{~mm}$ /an [20]. Les mois les plus arrosés sont janvier et novembre avec respectivement $43,8 \mathrm{~mm}$ et $44,3 \mathrm{~mm}$.

Tableau2. Régime pluviométrique saisonnier de la zone d'étude (1985-2015).

\begin{tabular}{|c|c|c|c|c|c|c|c|c|l|}
\hline \multicolumn{3}{|c|}{ Régimes saisonniers } & Type & $\begin{array}{c}\text { P } \\
\text { annuelles }\end{array}$ & M $\left({ }^{\circ} \mathrm{C}\right)$ & $\mathrm{m}\left({ }^{\circ} \mathrm{C}\right)$ & $\mathrm{Q} 2$ & $\begin{array}{c}\text { Etage } \\
\text { bioclimatique }\end{array}$ \\
\cline { 1 - 5 } $\mathrm{H}$ & $\mathrm{P}$ & $\mathrm{E}$ & $\mathrm{A}$ & & 357,1 & 33,8 & 1,37 & 34,37 & $\begin{array}{l}\text { Semi-aride } \\
\text { frais }\end{array}$ \\
\hline 117,9 & 103,54 & 29,1 & 106,54 & HAPE & & & &
\end{tabular}

Légende : $\mathbf{H}$ : Hiver, $\mathbf{P}:$ Printemps, $\mathbf{E}:$ Eté, $\mathbf{A}:$ Automne, $\mathbf{M}:$ Température moyenne du mois le plus chaud, $\mathbf{m}$ : Température moyenne du mois le plus froid. 
Le tableau 2 montre une répartition équitable des précipitations entre les trois saisons Hiver, Automne et Printemps avec une moyenne de $109.15 \mathrm{~mm}$. Pour sa part, le régime pluviométrique saisonnier est de type HAPE (Hiver, Automne, Printemps, Eté).

Pour leur part, les températures enregistrent également des fluctuations importantes avec un fort écart thermique entre l'Eté et l'Hiver. Le mois le plus froid est Janvier avec $4^{\circ} \mathrm{C}$ et le mois le plus chaud est Août avec $35^{\circ} \mathrm{C}$. Compte tenu de sa continentalité, la zone d'étude connait une forte abondance des gelées hivernales qui influencent sensiblement la baisse des températures avec une moyenne de 40 jours par an avec un maximum en Décembre et Janvier.

Les vents dominants sont ceux du nord-ouest en Hiver. Ils sont généralement très froids et humides. La saison sèche est caractérisée par des vents chauds et secs provenant des zones sud. On enregistre en moyenne 14 jours /an.

\subsection{Zonage écologique}

Nous avons procédé à un zonage écologique pour avoir une vision architecturale sur la disposition spatiale de la végétation, grâce aux relevées et à l'étude des échantillons. Quatre expositions dont quatre placettes ont été échantillonnées. Ces zones sont représentées par le type du sol, le climat, la composition floristique et la topographie. Chaque placette a été analysée minutieusement afin d'obtenir un maximum d'informations et d'établir une description rationnelle du fonctionnement des écosystèmes existants.

Les strates définies sont : arborée, arbustive et herbacée.

\subsection{Echantillonnage et choix des placettes}

La méthode utilisée pour caractériser les groupements à travers l'aire de répartition de ces espèces dans la région d'étude, comporte deux phases principales :

- Reconnaissance des peuplements par enquête préliminaire sur le terrain ;

- Choix des stations représentant des conditions écologiques différentes.

Les zones écologiquement homogènes qui en résultent, nous ont orienté le choix de l'emplacement des stations. A l'intérieur de ces zones, le choix des stations a été presque imposé, il est néanmoins orienté par la présence des formations à Tetraclinis articulata qui fait l'objet de cette étude.

Ces deux phases ont permis de caractériser quatre placettes représentatives pour chaque exposition (Nord, Est, Ouest et Sud).

\subsection{Type et forme des placettes}

Au niveau de chaque exposition, quatre unités circulaires avec un centre fixe et une superficie 
de 4 ares ont été installées. Au total, 16 placettes ont fait l'objet d'installation dans la région de Beni Affene. Chacune des placettes est délimitée par le biais de la mire de Parde et du viseur dioptrique du dendromètre Blum-Leiss [7]. Pour les placettes installées sur terrain en pente, la distance entre les deux voyants de la mire de Parde a fait l'objet de correction.

Les relevés ont été réalisés en fin d'Hiver et au Printemps, saison considérée comme optimale. Chacun de ces relevés comprend des caractères écologiques d'ordre stationnel, recensés ou mesurés sur terrain, notamment : le lieu, la date, l'altitude, l'exposition, la pente, la nature du substrat, la surface du relevé, le recouvrement et le type physionomique de la végétation.

\subsection{Etude du sol}

16 échantillons de sol ont été prélevés à une profondeur de $50 \mathrm{~cm}$ à l'aide de la tarière pédologique. Les paramètres mesurés sont : l'humidité, la granulométrie (pipette de Robinson), le $\mathrm{pH}$, la conductivité électrique, le taux de calcaire (calcimètre de Bernard) et le taux de matière organique.

\section{RESULTATS ET DISCUSSION}

\subsection{Etude des groupements de Tetraclinaie}

La végétation joue un rôle fondamental dans la structure et le fonctionnement de l'écosystème dont elle constitue une expression du potentiel biologique. Cependant, le couvert végétal naturel est soumis à un double stress édaphoclimatique d'une part et anthropozoogène d'autre part [8].

L'objectif de cette étude est de faire une sélection des espèces inventoriées pour en sortir une liste des espèces dites caractéristiques du Tetraclinis articulata ou encore les espèces végétales qui constituent les groupements à Thuya.

\subsubsection{Compositions systématiques}

Les inventaires floristiques effectués ont permis d'identifier 13 familles et 21 espèces avec une prédominance de la strate arborée (Cupressacées, Anacariacées, Fagacées, Oléacées) par rapport à la strate arborescente et herbacée (Globulariacées, Cistacées, Lamiacées, Liliacées, Fabacées, Poacées...etc.). 


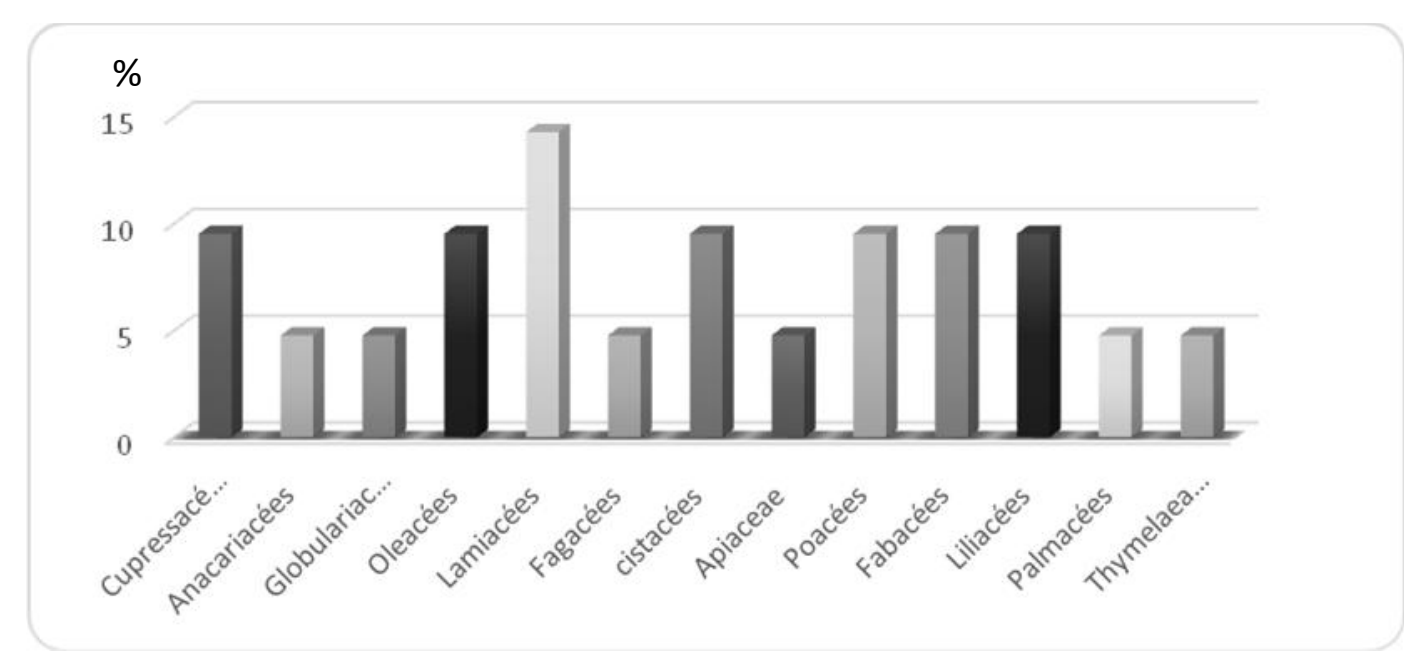

Fig.3. Pourcentages des différentes familles recensées dans la zone d'étude.

La répartition des familles dans la zone d'étude n'est pas homogène, les familles les mieux représentées sont: Lamiacées (14\%), Fabacées et Liliacées (10\%), Cupressacées (9\%), Cistacées (9\%), Oleacées et Globulariacées (9\%). Les autres familles : Thymelaeacées, Palmacées, Fagacées et Anacariacées représentées $5 \%$ (Figure 3).

Les familles dominantes dans le couvert végétal sont les plus riches en espèces inventoriées.

\subsubsection{Types biologiques}

La classification des différents types biologiques, fait apparaitre les catégories suivantes notamment les Phanérophytes, les Chamaephytes, les Géophytes, les Thérohytes et les Hémicryptophytes comme le montre la figure 4.

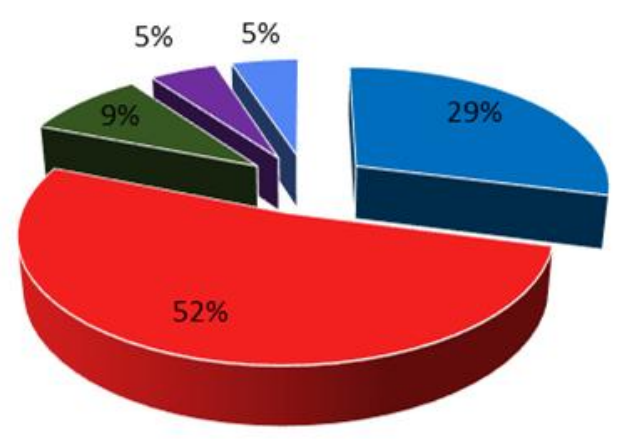

$$
\begin{aligned}
& \text { - } \mathrm{PH} \\
& \text { - } \mathrm{CH} \\
& \text { - } \mathrm{GE} \\
& \text { - } \mathrm{TH} \\
& \text { - } \mathrm{HE}
\end{aligned}
$$

Légende $: \mathbf{P h}:$ Phanérophytes, $\mathbf{C h}:$ Chamaephytes, Th : Thérophytes, Ge : Géophytes et He : Hémicryptophytes.

Fig.4. Pourcentage des différents types biologiques de la flore dans la zone d'étude.

La répartition des formations végétales par types biologiques est hétérogène. Elle est de type : $\mathrm{Ch}>\mathrm{Ph}>\mathrm{Ge}>\mathrm{Th}=\mathrm{He}$. 


\subsubsection{Classification des différents types morphologiques}

Du point de vu morphologique, les formations végétales sont marquées par l'hétérogénéité entre les ligneux et les herbacées, et entre les vivaces et les annuelles (tableau 3).

Tableau 3. Pourcentage des différents types morphologiques.

\begin{tabular}{|c|c|c|c|}
\hline Types morphologiques & LV(\%) & HA(\%) & HV(\%) \\
\hline Nord & 89 & 7 & 4 \\
\hline Ouest & 90 & 3 & 7 \\
\hline Sud & 97 & 0 & 3 \\
\hline Est & 91 & 9 & 0 \\
\hline Total zone d'étude & 67 & 19 & 14 \\
\hline
\end{tabular}

Légende :LV : ligneux vivaces, $\mathbf{H A}$ : herbacées annuelles et $\mathbf{H V}$ : herbacées vivaces.

Le type morphologique des ligneux vivaces sont les plus dominants avec un pourcentage de $67 \%$, par ailleurs, les herbacées annuelles sont en deuxième position avec $19 \%$, et enfin les herbacées vivaces avec $14 \%$.

Ces variations en pourcentage des types morphologiques peuvent s'expliquer par l'instabilité structurale du sol, et les rigueurs climatiques favorisent l'installation et le développement des espèces ligneuses vivaces, car elles résistent à différentes variations climatiques et édaphiques.

\subsubsection{Inventaire floristique}

L'inventaire floristique a permis de décrire une liste de 21 espèces caractéristiques du Tetraclinis articulata ou encore les espèces qui constituent les groupements de cette dernière (Figure 5). Par dominance, on distingue : Tetraclinis articulata $33 \%$, Rosmarinus tournefortii $15 \%$, Pistacia lentiscus $11 \%$, Phylleriaangustifolia $9 \%$, Globularia alypum $8 \%$, Cistussalviifolius $6 \%$, Stipa tenacissima et Olea europaea $5 \%$, Quercus coccifera $2 \%$ et Ferula communis $1 \%$. 


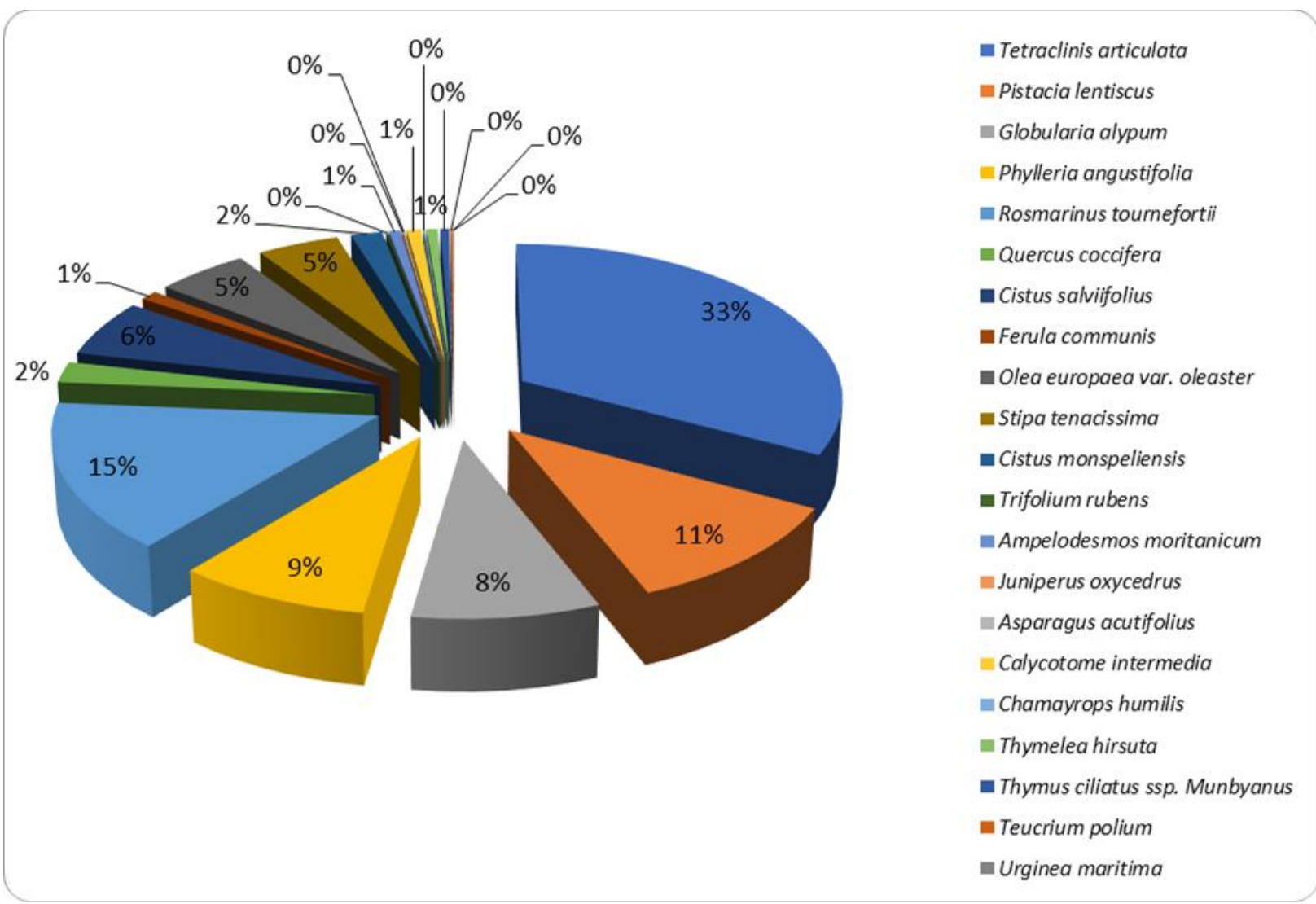

Fig.5. Résultats de l'inventaire floristique de la forêt de Beni Affene.

\subsection{Analyses physico-chimiques du sol}

La connaissance des aspects pédologiques permet de compléter les observations géographiques décrivant le milieu de croissance des essences. Ces informations permettent de caractériser l'état actuel du peuplement et les conditions écologiques dans lesquelles il se développe [9].

Les analyses physico-chimiques du sol réalisées au niveau de la zone d'étude ont permis de conclure ce qui suit :

L'humidité du sol est sensiblement stable dans les différentes expositions. Elle peut atteindre jusqu'à un seuil de $5 \%$. Ceci est dû à la faible teneur en argile qui induit une faible capacité de rétention.

La texture des sols dans les différentes expositions est dans l'ensemble limoneuse à limono- 
argileuse. Ce sont généralement des sols légers et perméables favorisant l'installation du Tetraclinis articulata. Cette espèce montre en effet, une résistance remarquable aux différents facteurs de dégradation et une faible exigence écologique vis-à-vis de la nature du sol et des précipitations. Tetraclinis articulata s'accommode à une texture dominée par les sables et les limons.

Pour ce qui est du pH, les échantillons analysés sont alcalins. Le pH varie entre 8,1 et 8,9. Nous ne remarquons pas de grande influence saisonnière sur le $\mathrm{pH}$ du sol. Kadik [10], rapporte que le Thuya peut s'accommoder à des sols à $\mathrm{pH}$ allant de 6,5 à 8,9 et que les meilleurs peuplements sont situés sur des sols à réaction basique dont le $\mathrm{pH}$ varie entre 7,5 et 8,5 à forte réserve calcique dans la terre fine [10].

Le taux de calcaire total est très élevé et peut atteindre jusqu'à $93 \%$. Le pourcentage de carbonate moyen se situe entre $42 \%$ et $73 \%$. En se référant à l'échelle de désignation des types de sols en fonction du taux du calcaire total [11], on peut dire que les sols sont fortement calcaires dans la plupart des stations étudiées. Ces résultats témoignent de l'adaptation des groupements à Tetraclinis articulata en s'installant sur un substrat à une teneur en carbonates assez élevée.

Le taux de matière organique est dans l'ensemble moyen pour l'ensemble des échantillons. Il varie entre $1,49 \%$ et $5,1 \%$. Duchaufour [12], considère qu'un sol est riche en matière organique au-delà d'un seuil de 2,0\% [12].

\subsection{Analyses phytoécologiques des groupements végétaux}

Dans ce contexte, nous avons réalisé une analyse factorielle des correspondances (AFC). Ce type de traitement a permis la mise en évidence de gradients écologiques pertinents intervenant dans la structuration de la végétation au niveau de chaque station. Trois modalités du milieu (exposition, type du sol, espèce) ont été prises en compte.

Pour chaque carte d'analyse factorielle, les pourcentages d'inertie expliqués par chacun des axes, ont été pris en considération. Les écarts relatifs nous ont permis de connaître le nombre des axes susceptibles d'être interprétés.

\subsubsection{AFC $\mathrm{N}^{\circ} 1$ : Exposition, placettes et espèces}

L'analyse des plans factoriels a permis l'identification de trois types de groupes $(1,2$ et 3$)$ de relevés grâce aux axes factoriels 1,2 .

\section{- Groupe 1}

Ce groupe renferme deux espèces de Chamaephytes : Rosmarinus tournefortii (RuA) et Thymelea hirsuta (TmP). Il est situé sur le côté positif de l'axe 1 et dans le côté négatif dans 
l'axe 2.

\section{- Groupe 2}

Ce groupe renferme deux espèces, une Chamaephyte: Teucrium polium (TcL), et une Phanérophytes : Olea europaea var. oleaster (OlP). Les espèces de ce groupe sont situées dans le côté positif des deux axes 1 et 2 .

\section{- Groupe 3}

Ce groupe englobe 17 espèces, cinq espèces de Phanérophytes : Tetraclinis articulata (Ta), Pistacia lentiscus (P1), Phillyrea angustifolia (PhL), Quercus coccifera (QC) et Juniperus oxycedrus (JoX), huit espèces de chamaephytes : Globularia alypum (Ga), Cistus salviifolius (CuS), Cistus monspeliensis (CsM), Ampélodesmos mauritanicum (Am), Asparagus acutifolius (AsP), Calycotome intermedia (CtI), Chamaerops humilis (CyH) et Thymus ciliatus ssp. munbyanus (ThA), deux espèces de Géophytes : Stipa tenacissima (St), Urginea maritima (Um), une espèce de Thérohytes: Trifolium rubens (TfR) et enfin une espèce d'Hémicryptophytes : Ferula communis (FcM).

Ces espèces sont localisées sur le côté positif de l'axe 1 à l'exception des espèces : Pistacia lentiscus (Pl), Phillyrea angustifolia (PhL), Trifolium rubens (TfR).

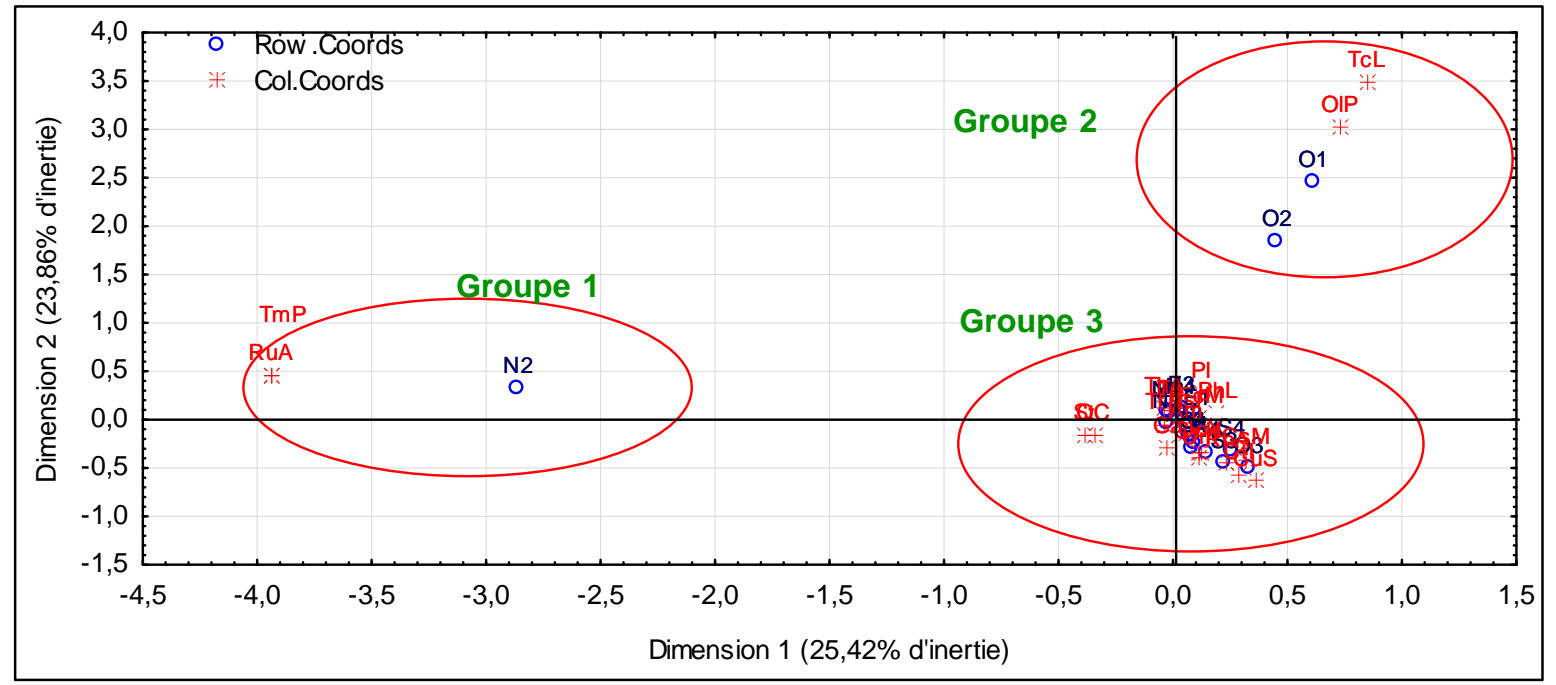

Fig.6. Plan factoriel des expositions et les placettes en fonction des espèces.

L'analyse des plans factoriels des expositions et les placettes en fonction des espèces illustrer dans la figure 6 , permet de définir, pour le premier groupe, que la placette $\mathrm{n}^{\circ} 2$ de l'exposition Nord (N2) contient un nombre très élevé en Rosmarinus tournefortii (RuA) avec un taux de recouvrement de $42 \%$. Cette espèce a un très bon pouvoir de conquérir les terrains dénudés. 
Elle est très commune dans l'aire de Thuya, vu sa fidélité qui est très élevée à cette espèce comme le témoigne Alcaraz [13].

Pour le deuxième groupe, nous remarquons que l'Olea europaea var. oleaster (O1P) est l'espèce la plus représentative dans les placettes 1 et 2 de l'exposition Ouest (O1 et O2) avec des taux de recouvrement de $52 \%$ et $40 \%$ respectivement. Malgré l'importance de cette dernière est l'une des espèces arbustives pré forestières liées au thuya qui est pratiquement le seul élément arborescent.

Cependant, sa densité en général importante, le Thuya n'arrive pas à couvrir entièrement le sol si bien que la structure physionomique dominante de l'association est celle de matorral arboré plus ou moins dense, en fonction de l'importance des arbustes pré forestiers tels que : Calycotome intermedia (CtI), Pistacia lentiscus (P1), Olea europaea var. oleaster (O1P) [14].

Concernant le troisième groupe, c'est le groupe qui renferme la majorité des espèces inventoriées. Il s'agit d'une formation pré forestière où le Tetraclinis articulata (Ta) est l'espèce dominante, accompagnée par Quercus coccifera (QC), Olea europaea var. oleaster (OlP), Juniperus oxycedrus (JoX), Ampélodesmos mauritanicum (Am), Calycotome intermedia (CtI) et Phillyrea angustifolia (PhL) [15].

Cette végétation constitue par la suite un milieu favorable aux incendies très souvent volontaires [17]. La présence des cistes indiquait que ces milieux ont été incendiés et indiqueraient des stades fortement dégradés [16].

\subsubsection{AFC $\mathbf{N}^{\circ} 2$ : d'Exposition- taux de recouvrement}

L'analyse des plans factoriels a permis l'identification de trois types de groupes de relevés grâce aux axes factoriels 1, 2 (figure 7).

\section{- Groupe 1}

Il renferme cinq espèces de Chamaephytes : Cistus salviifolius $(\mathrm{CuS})$, Chamayrops humilis (CyH), Calycotome intermedia (CtI), Thymus ciliatus ssp. Munbyanus (ThA) et Teucrium polium (TcL), et une Phanérophytes : Olea europaea var. oleaster (OlP). Ce groupe est situé sur le côté positif de l'axe 1 et 2 .

\section{- Groupe 2}

Il renferme cinq espèces, une Thérophyte: Trifolium rubens (TfR), une Phanérophytes : Quercus coccifera (QC), et trois Chamaephytes: Ampélodesmos mauritanicum (Am), Rosmarinus tournefortii (RhA) et Thymelea hirsuta (TmP). Les espèces de ce groupe sont situées dans le côté positif de l'axe 1 et dans le côté négatif du 2eme axe.

\section{- Groupe 3}


Le groupe 3 englobe 10 espèces dont quatre espèces de Phanérophytes : Tetraclinis articulata, Pistacia lentiscus (Pl), Phillyrea angustifolia ( $\mathrm{PhL}$ ) et Juniperus oxycedrus (JoX), trois espèces de Chamaephytes: Globularia alypum (Ga), Cistus monspeliensis (CsM) et Asparagus acutifolius (AsP), deux espèces de Géophytes : Stipa tenacissima (St), Urginea maritima $(\mathrm{Um})$, et une espèce d'Hémicryptophytes : Ferula communis (FcM). Ce groupe est localisé au centre des deux axes 1 et 2 .

On remarque au niveau de cet axe un gradient dynamique de végétation régressive du côté positif vers le côté négatif. On passe en effet des espèces Phanérophytiques, Chamaephytiques (Tetraclinis articulata (Ta), Calycotome intermedia (CtI)...etc.) aux espèces thérophytiques Trifolium rubens (TfR). Cet axe correspond à un gradient décroissant d'une évolution végétale.

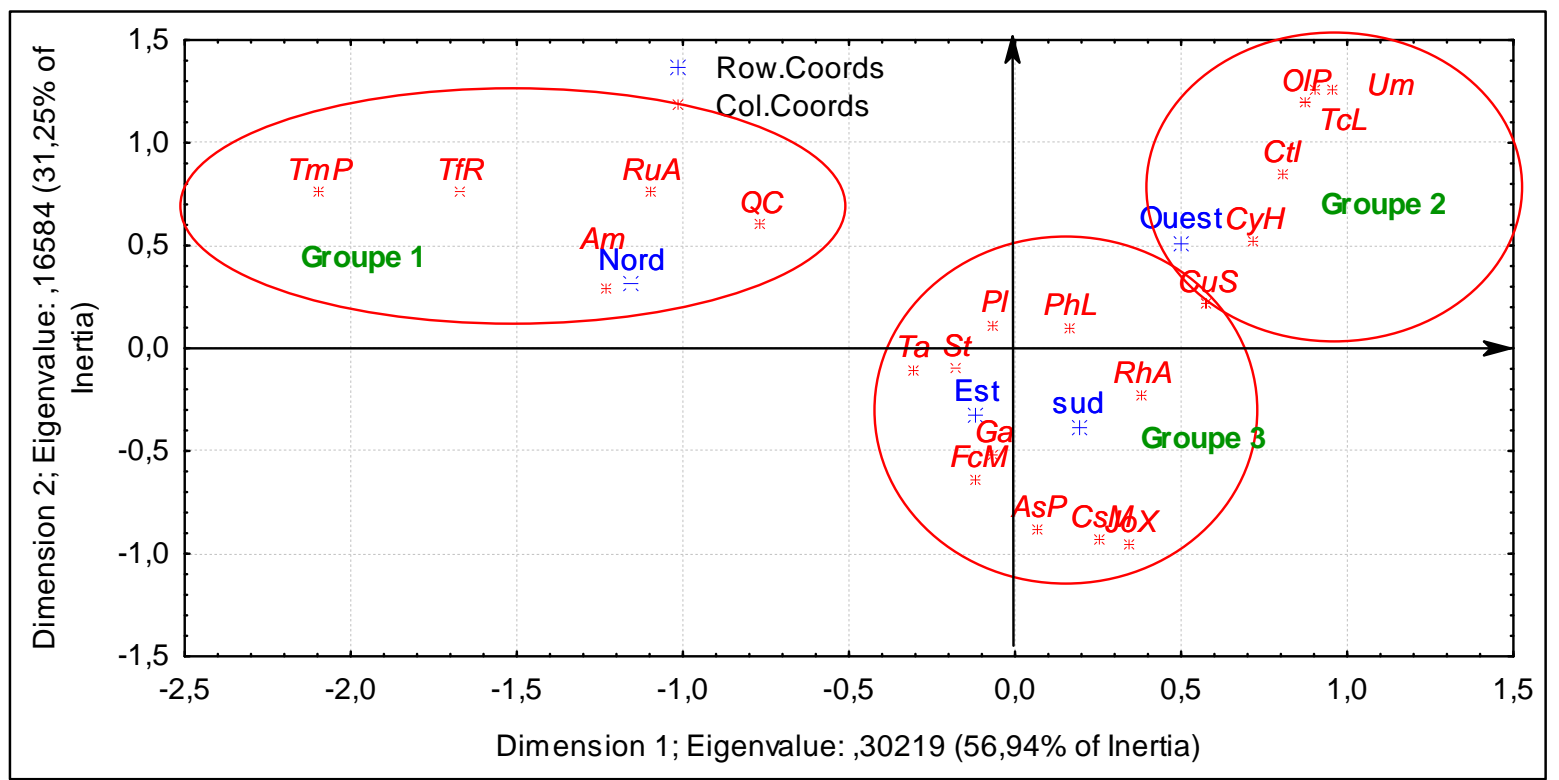

Fig.7. Plan factoriel des expositions en fonction du taux de recouvrement des espèces.

Le tapis végétal de la Tetraclinaie est dominé par des espèces parfaitement adaptées aux milieux secs et calcaires ; il s'agit de Stipa tenacissima (St), Ampélodesmos mauritanicum (Am), Thymus ciliatus ssp. Munbyanus (ThA), Rosmarinus tournefortii (RhA) et Globularia $\operatorname{alypum}(\mathrm{Ga})$.

\subsubsection{AFC $\mathrm{N}^{\circ} 3$ : Type du sol - espèces}

L'analyse type du sol - espèces montre que le taux d'inertie de l'axe 1 est élevé par rapport au taux d'inertie de l'axe $2(60,36 \%$ et $39,64 \%$, respectivement). Ceci montre que la projection se fait principalement sur le premier axe (figure 8). 
L'axe 1 oppose principalement deux groupes :

\section{- Côté positif}

Deux groupes d'espèces s'associent au type du sol. Le premier groupe renferme le sol argilolimoneux (AL) avec trois espèces : Ferula communis ( FcM), Cistus monspeliensis (CuM), Asparagus acutifolius (AsP), alors que le deuxième groupe englobe le sol limoneux (L) avec sept espèces (Ampélodesmos mauritanicum (Am), Globularia alypum (Ga), Stipa tenacissima (St), Cistus salviifolius (CuS), Phillyrea angustifolia (PhL), Tetraclinis articulata (Ta), Quercus coccifera (QC)).

\section{- Côté négatif}

L'axe $\mathrm{n}^{\circ} 1$ représente deux classes : du côté positif, l'extrémité de l'axe est constituée d'un groupe qui renferme trois espèces (Ferula communis (FcM), Cistus monspeliensis $(\mathrm{CuM})$, Asparagus acutifolius (AsP)) avec le sol argilo-limoneux (AL).

Le côté négatif s'individualise en deux groupes. Le premier groupe est constitué de sol limoneux (L) avec sept espèces (Ampélodesmos mauritanicum (Am), Globularia alypum (Ga), Stipa tenacissima (St), Cistus salviifolius $(\mathrm{CuS})$, Phillyrea angustifolia ( $\mathrm{PhL})$, Tetraclinis articulata (Ta), Quercus coccifera (QC)). Cependant le deuxième groupe associe le sol à limons fins (LF) avec les espèces suivantes : Chamaerops humilis $(\mathrm{CyH})$, Calycotome intermedia (CtI) et Olea europaea (OlP).

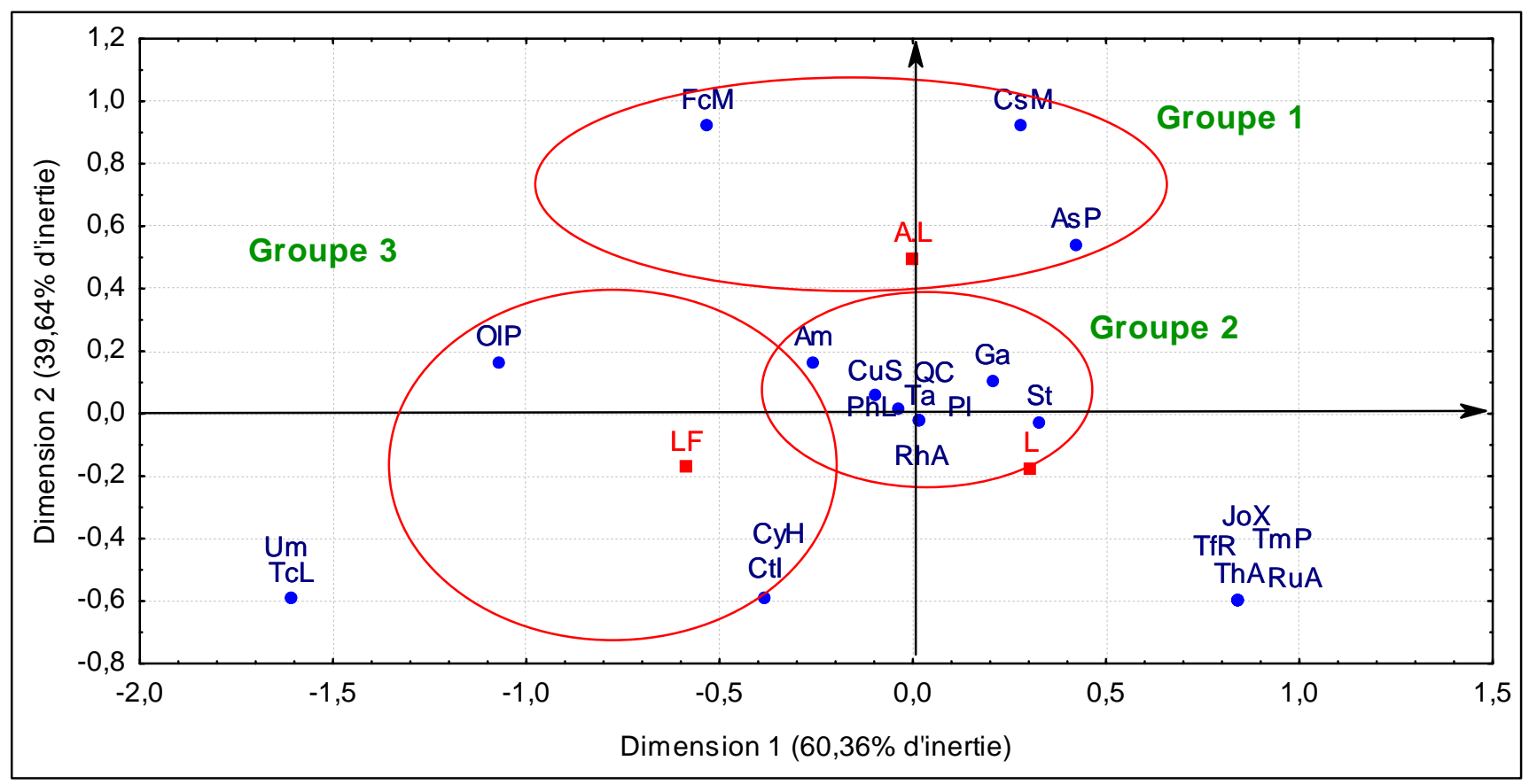

Fig.8. Plan factoriel entre le type de sol et les espèces. 
Gràce à sa plasticité et sa résistence écologique, l'espèce Tetraclinis articulata (Ta), peut coloniser des milieux sylvatiques, préforstiers et présteppiques. Elle joue, de ce fait, un rôle important dans la structure végétale de ces milieux là oû elle est présente [14].

\section{CONCLUSION}

Le massif forestier de Beni Affane a fait l'objet d'une étude phytoécologique des groupements à Tetraclinis articulata. Il a été choisi en raison de la dominance de l'espèce dans la région et sa résistance aux différents facteurs de dégradation.

L'étude bioclimatique de la région révèle un régime méditerranéen caractérisé par deux saisons bien distinctes : une période pluvieuse de cinq mois et une autre sèche plus longue, qui s'étend sur sept mois. Cette situation induit une forte évapotranspiration, ce qui lui permet de développer des systèmes d'adaptation modifiant ainsi le paysage en imposant une végétation xérophile.

$\mathrm{Du}$ point de vue végétation, il a été décrit une liste de 21 espèces caractéristiques du Tetraclinis articulata. Parmi lesquelles, 5 espèces sont fréquentes : Tetraclinis articulata, Pistacia lentiscus, Globularia alypum, phylleria angustifolia et Rosmarinus tournefortii.

L'analyse floristique de la zone d'étude a également permis de faire ressortir deux aspects importants :

Le couvert végétal est composé essentiellement par des espèces appartenant aux familles des Lamiacées, Liliacées, Fabacées, Cupressacées à concurrence de 51\%et d'autres familles moins riches en espèces ne représentent que de faibles taux ne dépassant guère les $5 \%$, telles que les Fagacées et les Thymelaeacées.

Le type biologique est représenté par des formations assez dégradées, marquées par une dominance des Chamaephytes, viennent en deuxième position les Phanérophytes, les géophytes, les Thérophytes et les Hémicryptophytes. Ces derniers exigent en effet un milieu riche en matière organique et une forte altitude.

Les principales essences qui imposent une dominance dans la composition floristique grâce à leur pouvoir de résistance aux diverses agressions, se résument au Tetraclinis articulata, pour les espèces forestières et Pistacia lentiscus, Phillyrea angustifolia pour les espèces préforestières.

Pour sa part, l'analyse factorielle des correspondances (AFC) a permis de considérer la végétation dans son dynamisme et sa physionomie, et de proposer des hypothèses quant à 
l'effet que peut jouer un certain nombre de facteurs écologiques notamment le bioclimat et l'action anthropique sur l'installation des formations végétales dans cette région.

\section{REFERENCES}

[1] Hadjadj Aoual S., Effet des facteurs environnementaux sur les premiers stades de la régénération naturelle de Tetraclinis articulata (Vahl, Master) en Oranie (Algérie). Ecologia mediterranea. 35, 2009, 20 - 31.

[2] Fennane M., Etude phytoécologique des Tetraclinaies marocaines. Thèse d'état. Univ. Aix Marseille III, 1987, 150 p.

[3] Hadjadj Aoual S., Les peuplements du thuya de berbérie en Algérie: phytoécologie Syntaxonomie, potentialités sylvicoles. Thèse Doct. Es. Sci. Univ. Aix - Marsaille, 1995,159p.

[4] Miloudi A., La régénération du Thuya de Berberie (Tetraclinis articulata), dans la forêt de Fergoug (Maroc). Thèse de magister. Instit. Nat. Agronomique. El Harrach. 1996, 150 p.

[5] CFT., 2015, Conservation des Forêts de la Wilaya de Tiaret. Service gestion : la répartition des formations forestières dans la Wilaya de Tiaret.

[6] Halitim A., Sols des régions arides d'Algérie.OPU. Alger, 1988, 384p.

[7] Parde J., Bouchon J., Dendrométrie. Nancy : École nationale du Génie rural, des Eaux et des Forêts, 1988, 328 p.

[8] Bouchetata T et Bouchetata A.,Dégradation des écosystèmes steppiques et stratégie de développement durable. Mise au point méthodologique appliquée à la Wilaya de Nâama (Algérie). Développement durable et territoires, Varia (2004-2010) |2005. 16 p.

[9] Lecomte H., Rondeux J., Les inventaires forestiers nationaux en Europe : tentative de synthèse. Les cahiers forestiers de Gembloux $\mathrm{N}^{\circ} 05,2002,29$ p.

[10] Kadik B., Contribution à l'étude du pin d'Alep (Pinus halepensis Mill) en Algérie, dendrométrie morphologie. Office des publications universitaires (OPU) Alger, 1987, 580 p.

[11] Baize D., Guide des analyses en pédologie. Ed. INRA, Paris,2000, 257p.

[12] Duchauffour Ph., Pédologie. Tome 1 : Pédogénèse et classification. Masson. Paris. 1977, $477 \mathrm{p}$.

[13] Alcaraz C., La végétation de l'ouest Algérien. Thèse Doct. Etat. Univ. Perpignan, 1982, $415 \mathrm{p}$.

[14] Fennane M., Tetraclinis formations of Morocco: Syntaxonomy and key to associations. Documents Phytosociologiques, 1988, 11 : 303-310. 
[15] Miara M. D., Hadjadj Aoul S., Ait Hammou M., Analyse phytoécologique et syntaxonomique des groupements végétaux dans le Massif de Guezoul-Tiaret (N-O Algérie). Bul. Soc. Bot. du Centre-Ouest.43, 2012, 279-316.

[16] Cherif I., Contribution à une étude phytoécologique des groupements à Tetraclinis articulata du littoral de Honaine (Algérie occidentale). Mémoire de magister, 2012, 167p.

[17] Benabadji N., Benmansour D., Bouazza M., La flore des monts d'Ain Fezza dans l'ouest algérien, biodiversité et dynamique. Sciences \& Technologie.26, 2007, 47-59.

[18] Barbero M., Bonin G., Loisel R., Quézel P., Sclerophyllus Quercus forests of the Mediterranean area. Ecological and ethological significance bieleferder. Beiter 4, 1989. Pp. 123.

[19] Pietracarprina P.,Morphologie et classification des sols CIHEAM (Bari), 1988, 96 p.

[20] ONM, 2015, Office National de la Météorologie, Service d'information météorologique.

How to cite this article:

Azzaoui M E, Maatoug M and Berrayah M. phytoecological study of tetraclinis articulata in the woodland of beni affene, sdamas chergui (Tiaret, Algeria). J. Fundam. Appl. Sci., 2017, 9(2), 971-987. 